\title{
Semantic and Pragmatic Context-Dependence: The Case of Reciprocals*
}

\author{
Yookyung Kim Stanley Peters \\ Stanford University
}

\section{Introduction}

This paper is about the meaning of reciprocal expressions in English. Each other and one another vary in meaning according to the meaning of their scope and antecedent, as well as the context in which they are uttered. Variation in the reciprocal's meaning is not just pragmatically determined alteration in speaker meaning but semantically determined change of literal conditions for strict truth. The dramatic range of observed variation is parameterized, and a principle is formulated which predicts the reciprocal's literal meaning in any context of utterance. We hypothesize that a reciprocal statement expresses the strongest candidate meaning that is consistent with certain contextually given information. This analysis explains a large collection of examples, including those with quantified antecedents.

The first two sections of this paper are descriptive, exploring a variety of examples to find whether their literal meaning changes or only the speaker's meaning. Sections 3 and 4 present parameters of variation in the meaning of the reciprocal and a generalization covering the meaning of each case considered. In Section 5, the Strongest Meaning Hypothesis is introduced and shown to give an analysis of the meaning of reciprocal expressions which explains observed shifts in their literal meaning. Section 6 spells out some surprising predictions that follow from the account proposed. The role of context in determining the meaning of the reciprocal expression is emphasized throughout the paper, and further contextdependency of the reciprocal is discussed in the final section.

\section{Variation in meaning of English reciprocal each other}

Research on the syntax and semantics of reciprocity often centers on sentences such as:

(1) John and Bill saw/kicked/laughed at each other.

Examples in which the reciprocal antecedent denotes a group with two members - here, John and Bill - are particularly easy to analyze. Usually when groups of two members are considered, each group member is required to stand in the stated relation to the other member.

Generalizing to larger antecedent groups, this suggests that

*We are indebted to Mary Dalrymple, Irene Hayrapetian, Ikumi Imani, Sam Mchombo, and especially to Makoto Kanazawa for helpful discussions of material in this paper. We also would like to thank participants at the conference of SALT V and LSA meeting in New Orleans for their valuable comments. 
(2) House of Commons etiquette requires legislators to address only the speaker of the House and refer to each other indirectly.

should mean that each legislator is required to refer to every other one indirectly, as the diagram (3) depicts. In each diagram in this paper, the set $A$ is the group of entities which comprise the domain determined by the antecedent of the reciprocal, and the arrows represent the relation which is determined by the scope of the reciprocal.

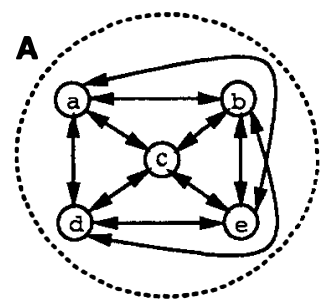

The diagram (3) shows that every House of Commons legislator has to refer to every other one only indirectly in order for statement (2) to be rue.

However, when reciprocal statements with antecedent groups larger than two members are considered extensively, ${ }^{1}$ these truth conditions turn out to be the wrong ones for many cases. ${ }^{2}$ Consider the following examples. For each statement, we give a diagram which depicts a typical situation described by it. Each sentence has different truth conditions, and we shall see that the variation is not random.

(4) As the preposterous horde crowded around, waiting for the likes of Evans and Mike Greenwell, five Boston pitchers sat alongside each other: Larry Andersen, Jeff Reardon, Jeff Gray, Dennis Lamp and Tom Bolton.

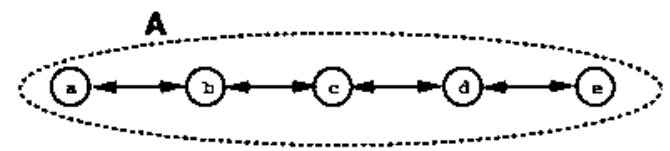

\footnotetext{
${ }^{1}$ We examined 1740 examples from the Hector Corpus and 1579 examples from the New York Times. The authors are grateful to Oxford University Press for permission to study and use citations from the Oxford 'Hector' Corpus, a pilot corpus for the British National Corpus which was used for 'Hector' a research project in lexical computing by the Systems Research Center of Digital Equipment Corporation and the Dictionaries Department of Oxford University Press.

${ }^{2}$ We are not concerned here with reciprocal statements like (i), in which the scope relation must hold between groups rather than entities in the domain.

(i) The satellite, called Windsock, would be launched from under the wing of a B-52 bomber and fly to a "libration point" where the gravitational fields of the Earth, the Sun and the Moon cancel each other out.
}

The meaning of basic reciprocal statements can and should be characterized independently of such sentences, which are limited in use. 
It is obvious that the reciprocal in (4) has a different meaning than that in (2). Its truth conditions are weaker, it does not require every member to be related to every other member by the relation of sitting alongside. The next pair of examples have truth conditions even weaker than this: the relation holds asymmetrically, as represented by single-headed arrows in the diagram, in contrast with the double-headed arrows in diagrams above. If one individual is related to another, the second is not related to the first by giving measles to or being stacked on top of.

(6) Mrs. Smith's third-grade students gave each other measles.

(7) They climbed a drainpipe to enter the school through a high window and stacked tables on top of each other to get out again.

(8)

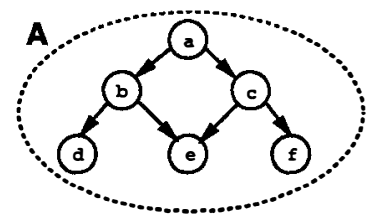

Statement (9) exemplifies another meaning of the reciprocal: every member is claimed to relate to at least one other and, unlike examples (6) and (7), being passively related is not sufficient.

(9) "The captain!" said the pirates, staring at each other in surprise.

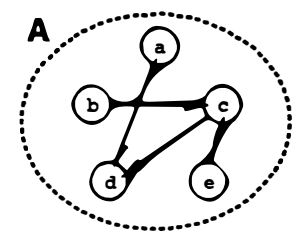

In statement (11), the reciprocal means something still different, allowing for multiple stacks of planks as compared with a single stack of tables.

(11) $\mathrm{He}$ and scores of other inmates slept on foot-wide wooden planks stacked atop each other - like sardines in a can - in garage-sized holes in the ground.

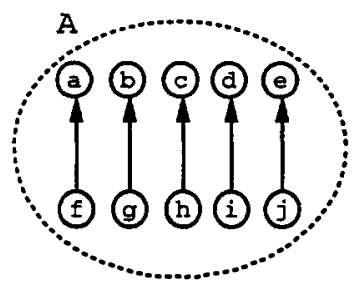


In all examples thus far, the antecedent denotes a group, which is taken as the domain of the reciprocal. Our data also include many examples with quantified antecedents. These exhibit variation in meaning of the reciprocal similar to what we have seen so far. One difference to note in the case of reciprocals with quantified antecedents is that in general the reciprocal is not claimed to hold of the whole group $A$ but rather of a (largest closed) subgroup $X$ of it. We will discuss this in detail later on. For now, please focus in the following examples on variation in the reciprocal's meaning.

First, (13) and (14) are the case where every member of the relevant set relates to every other member, similar to example (2).

(13) Discussions can get heated at times, but we try to keep them light-hearted and most women welcome the chance to hear and understand each other's points of view.

(14) Accounts of watching football composed by ordinary spectators emphasize how familiar many people were to one another, the wide age-range, and the lively banter. You knew everybody. You never saw 'em between games. But we always stood roughly in the same place and we knew the forty or fifty people around us 'cos they were always there.

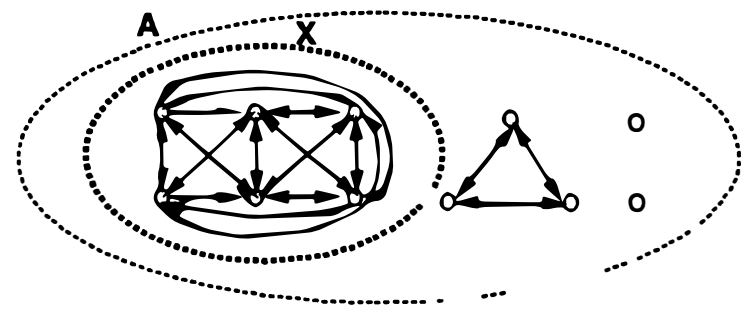

On the other hand, (16) has similar ruth conditions to (4), requiring that every one of the many vertebrae be indirectly or directly related to every other one.

(16) The number of vertebrae varies from 39 to 63 (the larger number in longnecked species), with many in the trunk fused to each other and to other bony elements to form a rigid central framework for flight.

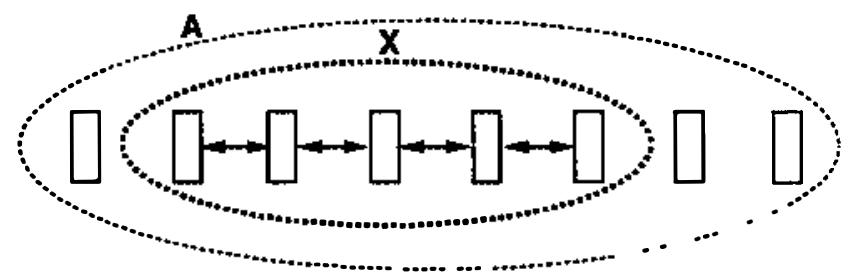

The statement

(18) Many people at the party yesterday are married to each other. 
has notably weaker truth conditions, as shown below. It does not claim anyone is married to more than one person.

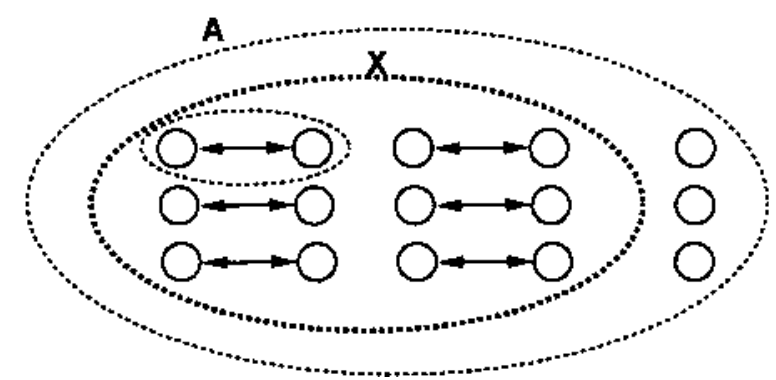

\section{Variation of Literal Meaning or just Speaker's Meaning?}

It is well known that what a speaker means to communicate can differ in certain respects from the literal meaning of his or her utterance. The speaker's meaning can be stronger in some ways than the literal meaning due to factors such as Grice's Cooperative Principle, which entitles speakers to count on hearers to recognize utterances' conversational implicatures not entailed by the literal meaning. Someone who says

(20) John is doing quite well in his new job working at the bank; he likes his colleagues, and he hasn't been to prison yet.

is ordinarily entitled to expect her hearer to understand she means John is the sort of person likely to yield to the temptation provided by working in a place with lots of money around.

On the other hand, the speaker's meaning can also be weaker in some ways than the literal meaning due to factors such as the speaker's indicating an intention not to be held strictly accountable for the truth of what he or she said but to be understood rather as describing a situation somewhat loosely. As Austin pointed out, a general can truthfully state

(21) France is hexagonal.

in discussing strategy for defense of the country's frontiers. But if a geometer were to assert (21), his or her statement would be false. Undulations in the 'sides' of French territory is irrelevant to the ruth of the general's loose statement, but not the geometer's strict one.

The following contradictory statement shows that the strong meaning of the reciprocal in (2) does not come from pragmatic strengthening, but is rather a genuine part of the reciprocal's conventional meaning.

(22) \# House of Commons legislators refer to each other indirectly; the most senior one addresses the most junior one directly.

Note in contrast the felicity of the following example: 
(23) House of Commons legislators refer to each other indirectly, except the most senior one addresses the most junior one directly.

In general, the exception construction is felicitous only in the presence of universal or negative universal quantification over appropriate $n$-tuples. ${ }^{3}$ Witness the following contrast:

(24) a. Every legislator refers indirectly to every other legislator, except the oldest one addresses the youngest directly.

b. \# Every legislator refers indirectly to some other legislator, except the oldest one addresses the youngest directly.

c. Every legislator refers indirectly to some other legislator, except the oldest one addresses some other one directly.

Nevertheless, the statement

(25) Five Boston pitchers sat alongside each other; Larry Andersen and Tom Bolton sat on the ends, separated by Jeff Reardon, Jeff Gray and Dennis Lamp.

is felicitous, showing a clear contrast to (22). Explicitly acknowledging that there are two pitchers (Larry Andersen and Tom Bolton) who are not sitting next to each other does not make the sentence contradictory. Notice that arbitrary relaxations of the stringent truth conditions of Strong Reciprocity do not preserve the truth of the utterance; sentence (4) would not be judged true in the following situation, where non-pitchers intervene among the pitchers:
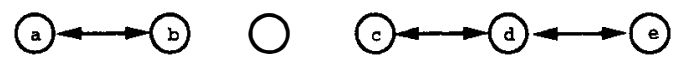

Likewise, the fact that the following statement is contradictory shows that the meaning of the reciprocal in (7) cannot be arbitrarily weakened.

(27) \# The tables are stacked on top of each other; one of them is sitting by itself. The existence of a table that is not stacked on any other one and has no table stacked on it is sufficient to falsify the claim (7).

Sentence (28) is similarly contradictory, as the second clause contradicts the first clause, which asserts that every pirate is staring at some other pirate.

(28) \# The pirates were staring at each other in surprise; one of them wasn't staring at any pirate.

Truth of the different reciprocal statements depends on meeting conditions of varying strength. These data show it would not be adequate to postulate some fixed, weak truth conditions for the reciprocal (e.g., what Langendoen (1978) called Weak Reciprocity) and rely on pragmatic strengthening to give a stronger speaker's meaning in cases where we have seen the speaker really means something stronger. Each of the reciprocal statements we have considered is literally false if the stronger

\footnotetext{
${ }^{3}$ See Moltmann (1993) although she suggests that universal quantification over pairs may be only implied, not asserted, by reciprocal sentences like (2).
} 
conditions are not met; however, that would not be so in the case of pragmatic strengthening by means, e.g., of conversational implicature.

What about the converse strategy: relying on pragmatic weakening to loosen some fixed, strong truth conditions we might uniformly assign, say Strong Reciprocity, thereby yielding a weaker speaker's meaning in cases where that is what we observed? This would not be adequate either, because as we pointed out the statements are strictly true even in conditions falling far short of those required by Strong Reciprocity.

Thus the literal meaning of the reciprocal really does vary across these statements. We must seek a semantic explanation of how it varies; we cannot claim the literal meaning is fixed and only the speaker's meaning varies.

\section{Reciprocal Expresses a Polyadic Quantifier}

Let us point out something that, though implicit in the literature on reciprocals, was only made explicit in Dalrymple et al. (1994):

- Reciprocal expressions such as English each other and one another, Chicheŵa -an, Japanese (o)tagai and Korean selo express polyadic quantifiers that bind two variables from their scope, both variables ranging over a specified set (the restricted domain of the quantification).

- $\operatorname{RECIP}(A, R)$

Thus a sentence like

(29) Tom, Dick and Harry saw each other.

expresses a proposition that might be symbolized

(30) $\operatorname{RECIP}(\{$ Tom, Dick, Harry $\}, \lambda x y . x$ saw $y)$

using the quantifier symbol RECIP, with its restricted domain $\{$ Tom, Dick, Harry $\}$ and its scope.

RECIP is a generalized quantifier of type $\langle 1,2\rangle$. The meaning of this quantifier is a relation between sets $A$ of members of the universe of discourse and two-place relations $R$ on the universe of discourse (van Benthem, 1989). A formula

$$
\operatorname{RECIP}(A, \lambda x y . \phi)
$$

is true iff the relation RECIP holds between the set $A$ and the binary relation of which $\lambda x y . \phi$ is the characteristic function.

Our purpose will be to propose a definition for RECIP, illustrating our claims with sentences taken from corpora we have examined. Most of the proposed meanings in previous literature (section 3.1) can be stated in terms of a set of simple relations. This allows for a specification of entailments among these meanings. We will make crucial use of these entailments in our definition of RECIP, presented informally in Section 5.

\subsection{Parameterizing the Reciprocal's Literal Meaning}

Langendoen (1978) and Kański (1987) proposed the following definitions of reciprocity. 


\section{Strong Reciprocity}

(SR) $|A| \geq 2$ and $\forall x, y \in A(x \neq y \rightarrow R x y)$

SR is the reciprocal's meaning in examples (1), (2), (13) and (14).

Symmetric Reciprocity

(SmR)

$$
\forall x \in A \exists y \in A(x \neq y \wedge R x y \wedge R y x)
$$

\section{Intermediate Reciprocity}

(IR) $|A| \geq 2$ and

$$
\forall x, y \in A(x \neq y \rightarrow
$$

for some sequence $z_{0}, \ldots, z_{m} \in A\left(x=z_{0} \wedge R z_{0} z_{1} \wedge \cdots \wedge R z_{m-1} z_{m} \wedge z_{m}=y\right)$

IR is the reciprocal's meaning in examples (4) and (16).

\section{Inclusive Alternative Ordering}

$$
\forall x \in A \exists y \in A(x \neq y \wedge(R x y \vee R y x))
$$

IAO is the reciprocal's meaning in examples (11) and (18).

To understand the logical relations among the various definitions, Dalrymple et al. (1994) analyzed $\operatorname{RECIP}(A, R)$ as $Q^{\sharp}\left(A, R^{\dagger}\right)$, where $Q^{\sharp}$ is FUL $\backslash I, \operatorname{LIN} \backslash I$ or TOT $\backslash I,{ }^{4}$ following a suggestion of Dag Westerståhl (p.c.):

$$
\begin{array}{rll}
\operatorname{FUL}(A, R) & \stackrel{\text { df }}{\leftrightarrow} R\lceil A=A \times A \\
\operatorname{LIN}(A, R) & \stackrel{\text { df }}{\leftrightarrow} R^{+}\lceil A=A \times A \\
\operatorname{TOT}(A, R) & \stackrel{\text { df }}{\leftrightarrow} \operatorname{dom}(R \uparrow A)=A \\
\operatorname{FUL} \backslash I(A, R) & \stackrel{\text { df }}{\leftrightarrow} \operatorname{FUL}(A, R \cup I) \\
\operatorname{LIN} \backslash I(A, R) & \stackrel{\text { df }}{\leftrightarrow} \operatorname{LIN}(A, R \cup I) \\
\operatorname{TOT} \backslash I(A, R) & \stackrel{\text { df }}{\leftrightarrow} \operatorname{TOT}(A, R \backslash I)
\end{array}
$$

$R^{\dagger}$ is either $R \uparrow A$ or the result of applying to it one of two operations: ${ }^{\wedge}$ and ${ }^{\vee}$.

$$
\begin{array}{lll}
R^{\wedge} & \stackrel{\mathrm{df}}{=} & R \cap R^{-1} \\
R^{\vee} & \stackrel{\mathrm{df}}{=} & R \cup R^{-1}
\end{array}
$$

Three of Langendoen's six definitions and one of Kański's two new ones are expressible in these terms. Partitioned Strong Reciprocity, Partitioned Intermediate Reciprocity, Weak Reciprocity ${ }^{5}$ and Kański's Exclusive Altemative Ordering are not definable in these terms. Despite earlier claims, we are aware of no examples that unequivocally attest any of these definitions. ${ }^{6}$ The given format of defining

\footnotetext{
${ }^{4}$ Actually, the analysis presented here improves on that in Dalrymple et al. (1994), enabling us to solve a problem not resolved there about the interpretation of sentences like (6) and (7).

${ }^{5}$ Weak Reciprocity can be expressed as follows, using conjunction and the relational converse: TOT $\backslash I(A, R) \wedge$ TOT $\backslash I\left(A, R^{-1}\right)$.

${ }^{6}$ See Dalrymple et al. (1994) for more detailed discussion.
} 
RECIP gives rise to 8 non-equivalent possible definitions, of which four are new possibilities that have not been considered in the literature: [1], [2], One-way Weak Reciprocity (OWR), and Intermediate Altemative Reciprocity (IAR).

The following graph depicts the implications among these definitions:

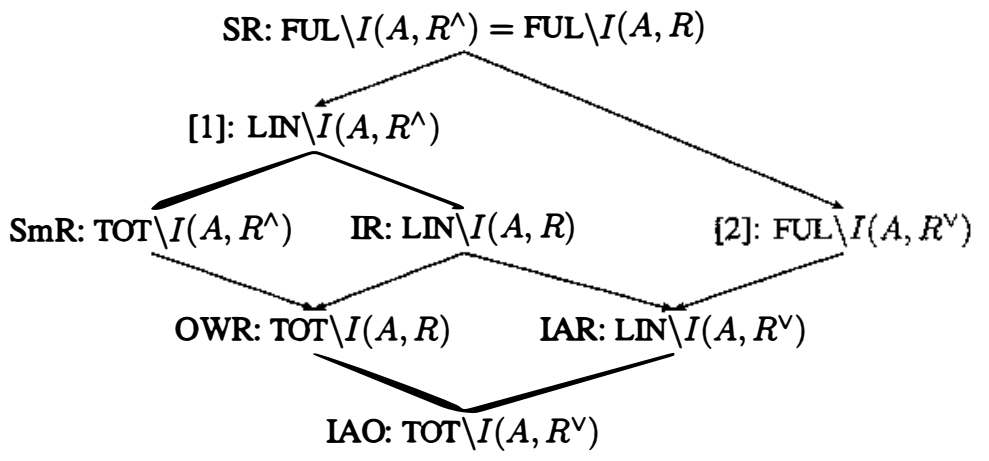

\subsection{Dependence of Partial Ordering on Properties of Restricted Scope Rela- tion}

Under certain circumstances, some of the definitions discussed in the previous section become equivalent. In case the scope relation $R$ is transitive and symmetric, for example, the nine possible reciprocal meanings collapse to only two separate cases. Care must be taken to control for these factors in considering evidence for the potential reciprocal meanings under discussion.

Symmetric $R$ : If $R$ is symmetric, $R=R^{-1}=R^{\wedge}=R^{\vee}$ and the partially ordered possibilities reduce to:

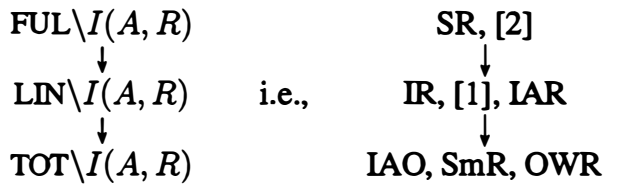

Transitive $R$ : If $R$ is transitive, $R=R^{+}$and the partially ordered possibilities reduce to:

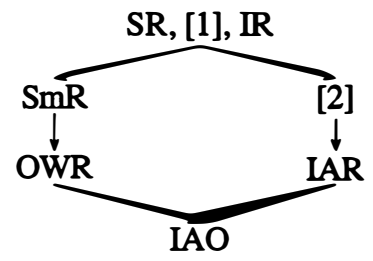

Transitive and symmetric $R$ : If $R$ is transitive and symmetric, the partially ordered possibilities reduce to:

$$
\begin{aligned}
& \text { FUL } \backslash I(A, R), \operatorname{LIN} \backslash I(A, R) \quad \text { i.e., } \quad \mathrm{SR}, \mathbb{R} \text {, [1], [2], } \mathrm{LAR} \\
& \text { TOT } \backslash \text { I }(A, R) \\
& \text { IAO, SmR, OWR }
\end{aligned}
$$


Asymmetric $R$ : If $R$ is asymmetric, $R^{\wedge}=\emptyset$ and SR, [1], and $\mathrm{SmR}$ are necessarily false (assuming $|A| \geq 2$ ).

\section{Generalization}

In the first two sections of this paper, we showed that there is variation in meaning of the reciprocal. In the third section, we showed how to analyze this along two dimensions of variation. Given our parameterization of the reciprocal's meaning, and the partial ordering by entailment of the resulting interpretations, we can see an empirical generalization lying behind the examples we discussed. We start from the simpler case - reciprocal sentences with group-denoting antecedents, and then deal with those with quantified antecedents (which involve the added complication of analyzing how to combine the antecedent quantifier and the RECIP quantifier). The examples with quantified antecedents are crucial, for they will show that the generalization derived for the meaning of the reciprocal in section 4.1 indeed holds of the combination of the antecedent and the reciprocal with its scope. Specifically, the parameters of reciprocal meaning are set so that reciprocal statements, not just the reciprocal expression, has the strongest possible candidate meaning.

\subsection{Reciprocals with group denoting antecedents}

(35) (=(2)) House of Commons legislators refers to each other indirectly.

The reciprocal in statement (35) is interpreted as Strong Reciprocity, which is the strongest candidate meaning in the partial ordering, and that interpretation is not contradicted by any contextually given information.

(36) (=(4)) As the preposterous horde crowded around, waiting for the likes of Evans and Mike Greenwell, five Boston pitchers sat alongside each other: Larry Andersen, Jeff Reardon, Jeff Gray, Dennis Lamp and Tom Bolton.

The reciprocal in statement (36) is interpreted as $I R$. The meaning of the words in this sentence, together with the nonlinguistic fact that people have only two sides, is inconsistent with any stronger truth conditions than IR. The reciprocal's scope in (36) is the symmetric relation of sitting alongside. So the 8 truth conditions under discussion collapse to 3: $\mathrm{SR}(=[2]) ; \mathrm{IR}(=[1], \mathrm{IAR})$; and $\mathrm{IAO}(=\mathrm{SmR}, \mathrm{OWR})$. When the group $A$ of pitchers consists of more than three members, SR cannot hold, as people have only two sides. Of course, IAO is consistent with the properties of sitting alongside; but so is the logically stronger $\mathbb{R}$, which is what the reciprocal in statement (36) means.

(37) (=(7)) They climbed a drainpipe to enter the school through a high window and stacked tables on top of each other to get out again.

The relation of stacking on top of in (37) is necessarily asymmetric, so SR, $\mathrm{SmR}$ and [1] cannot hold. If there are finitely many tables, OWR entails that a table on the bottom is stacked on top of a table above, which is impossible. [2] entails that every pair of the tables is in direct physical contact with each other, which is possible only if there are exactly two tables, in which case [2] is equivalent to IAR and IAO. This leaves only IAR and IAO as consistent interpretations of (37). IAR 
entails there is one stack, while the weaker condition IAO allows multiple stacks. In the given context, it is clear that one stack is the right interpretation, i.e., the reciprocal in statement (37) means IAR.

In each case, we see that the reciprocal is interpreted as having the logically strongest candidate meaning which is consistent with the meanings of the reciprocal's scope and antecedent as well as with relevant nonlinguistic information.

\subsection{Reciprocals with quantified antecedents}

In the case of reciprocal sentences with group denoting antecedents, the antecedent NPs directly provides with the set which is the first argument of the polyadic quantifier RECIP. However, Reciprocal sentences with quantified antecedents such as (36) and (37)

(38) (=(13)) Discussions can get heated at times, but we try to keep them lighthearted and most women welcome the chance to hear and understand each other's points of view.

(39) (=(14))Accounts of watching football composed by ordinary spectators emphasize how familiar many people were to one another, the wide age-range, and the lively banter. You knew everybody. You never saw 'em between games. But we always stood roughly in the same place and we knew the forty or fifty people around us 'cos they were always there.

involve two quantifiers: the type $\langle 1\rangle \mathcal{Q}$ expressed by the antecedent NP and formed from the determiner $Q$ of type $\langle 1,1\rangle$ and the VP $\mathcal{P}$ formed from the polyadic quantifier RECIP of type $\langle 1,2\rangle$.

$$
\begin{aligned}
& \mathcal{Q}=\{X \mid Q(A, X)\} \\
& \mathcal{P}=\{X \mid \operatorname{RECIP}(X, R)\}
\end{aligned}
$$

For instance, the two quantifiers in statement (39) are as follows:

$$
\begin{aligned}
& \mathcal{Q}=\{X \mid \operatorname{MANY}(\text { PEOPLE }, X)\} \\
& \mathcal{P}=\{X \mid \operatorname{RECIP}(X, \lambda x y . x \text { is familiar with } y)\}
\end{aligned}
$$

The question is: how are these meanings composed to yield the meaning of a reciprocal sentence with a quantified antecedent? We propose the following mode of combination for the two quantifiers.

\section{Bounded Composition}

$$
\text { (BC) } \begin{aligned}
\exists X \subseteq A(Q(A, X) \wedge \operatorname{RECIP}(X, R) \wedge \\
\forall Y \subseteq A(\operatorname{RECIP}(Y, R) \rightarrow((X \subseteq Y \rightarrow X=Y) \wedge(|X| \leq|Y| \rightarrow|X|=|Y|)))) \vee \\
(Q(A, \emptyset) \wedge \neg \exists X \subseteq A \operatorname{RECTP}(X, R))
\end{aligned}
$$

where $|X|$ denotes the cardinality of the set $X$, i.e., the requirements of $Q$ are met by a largest closed group of $A$ s of which RECIP holds for $R$, unless $Q(A, \emptyset) \wedge \neg \exists X \subseteq$ $A \operatorname{RECIP}(X, R) .^{7}$

\footnotetext{
${ }^{7}$ This rule is not strictly compositional; we need separate reference to the denotation of the common noun subphrase of the antecedent NP, i.e. the set $A$.
} 
Bounded Composition composes two quantifiers to produce the proposition that the requirements of the antecedent NP's determiner are met by a largest closed group of which RECIP holds for the scope relation in question, unless there is no such set. ${ }^{8}$

The particular statement in (39) does not require as complicated a combination as (BC): the statement would be true iff there is a subset $X$ containing most members of $A$ 's and RECIP holds of that set $X$ for the relation of being familiar with. In general, only the first conjunct of $(\mathrm{BC})$ is necessary for the ruth conditions of reciprocal statements which have monotone increasing quantifiers as their antecedents. But, when the antecedent quantifier is non-monotone, as in (40), the mere existence of a subset of which RECIP holds is not a sufficient condition.

(40) Exactly five men hit each other.

The second conjunct of (BC) states that the set $X$ of which RECIP holds for the relation of hitting should be a complete cluster (i.e., if another element were added to $X$, the resulting set would not satisfy RECIP, as $X$ does). Without this condition, (40) would be judged true if 7 men hit each other, since we could then choose a subset small enough to satisfy the quantifier exactly five. But with the complete cluster condition, the complete set (in the given situation, the set of 7 men hitting each other) rather than an arbitrary subset of the set must be selected; thus the statement (40) is correctly judged false. When there are more than one complete cluster of the relevant relation, (BC) seeks the largest set among them. ${ }^{9}$

On the other hand, Higginbotham (1980) proposed quantification over groups to deal with reciprocals with quantified antecedents, only considering quantifiers like some, all, no. As Roberts (1987) noted, his analysis would give wrong ruth conditions for quantifiers like most or many: (38) can be true even though most groups of women do not satisfy the reciprocal relation, and (39) can be false even though many groups of people satisfy the reciprocal relation. In our account, quantified antecedents of reciprocals quantify over individuals, not over groups.

Let us discuss what kind of RECIP is involved in each case. The reciprocal in the statement (39) is interpreted as Strong Reciprocity; (39) is true iff there is a set $X$ of many people such that every person in $X$ is familiar with every other one. This is the truth conditions when the antecedent quantifier is combined with Strong Reciprocity by Bounded Composition. Similarly, the statement (38) is true iff a largest closed set of women of which Strong Reciprocity holds for the relation of welcoming the chance to hear each other's points of view consists of most women in the domain. Recall that the diagram in (13) contains two closed groups of which Strong Reciprocity holds. Between those two, the largest group is chosen by Bounded Composition, and if that group consists of most women in the domain, the statement is judged true.

\footnotetext{
${ }^{8}$ The last proviso is necessary only for monotone-decreasing quantifiers: a statement with a monotone-decreasing quantifier should come out true even if there is no set of which RECIP holds for the given scope.

${ }^{9}$ Roberts (1987) proposed a similar semantics for reciprocals with quantified antecedents which considers a set of largest cardinality, treating determiners as cardinal adjectives.
} 
While the partial ordering in (31) shows that Strong Reciprocity is the strongest meaning, can it be still the strongest when it combines with another quantifier by Bounded Composition? The answer is positive, if the antecedent quantifier is monotone-increasing.

Fact 1. For monotone increasing $Q$, if $\mathrm{RECIP}_{1} \Rightarrow \mathrm{RECIP}_{2}$, then $(\mathrm{BC})\left[Q, \mathrm{RECIP}_{1}\right] \Rightarrow(\mathrm{BC})\left[Q, \mathrm{RECIP}_{2}\right]$.

Fact 1 saids that with a monotone increasing quantifier such as many, the logical relation among various RECIPS is preserved. Thus, the statements in (38) and (39, whose reciprocals are interpreted as SR, have the strongest possible interpretation.

(41) $(=(16))$ The number of vertebrae varies from 39 to 63 (the larger number in long-necked species), with many in the trunk fused to each other and to other bony elements to form a rigid central framework for flight.

The relation of being fused to is symmetric, so only three definitions of RECIP are distinguishable in the case of statement (41). The non-linguistic knowledge that vertebrae are arrayed along a line contradicts the possibility that any set of more than two vertebrae could satisfy $\operatorname{SR}(=[2])$, and two is too few to constitute many. So only $\mathbb{R}(=[1], \mathrm{IAR})$; and and $\mathrm{IAO}(=\mathrm{SmR}, \mathrm{OWR})$ yield consistent interpretations of statement (16). IR is the stronger interpretation, in light of Fact 1 ; and $\mathbb{R}$ is what the reciprocal means in (41).

The statement

(42) (=(18)) Many people at the party yesterday are married to each other.

does not mean that there is a set of many party goers each two of whom are married, nor even each two of whom are connected by a marriage chain. It means rather that the total number of party goers whose spouse is at the party is many. In the diagram (19), what must contain many party goers is the big dotted circle $X$, which includes all couples. That set does not satisfy $\mathrm{SR}(=[2])$ or $\mathbf{I R}(=[1],[3])$. Indeed, our cultural assumptions in monogamous society do not accept that a set with more than two people satisfies $S R$ or $\mathbb{R}$ for the relation of marrying. The one remaining definition $\operatorname{SmR}(=\mathrm{IAO}$, [4],WR) of reciprocal, however, is closed under union; so SmR holds of the sum of all couples. And this total sum is the largest set the mode (BC) seeks, 
as Fact 2 states. ${ }^{10}$

Fact 2. If RECIP is definable using TOT $\backslash I$ and $R$ is symmetric, then (BC) $\Leftrightarrow$ $Q(A,\{x \mid \exists y \in A(x \neq y \wedge R x y)\})$.

Therefore, the proposed mode and an appropriate RECIP nicely accounts for the interpretation.

\section{The Strongest Meaning Hypothesis}

What does a reciprocal sentence $S$ express in a context $c$ ?

Meaning of $S$

Context $c \longrightarrow$ Logically strongest $p$ instantiating (31) consistent with assumptions in $c$ as well

More specifically, as the meaning of the words in S.

(SMH) A reciprocal sentence $\mathrm{S}$ can be used felicitously in a context $c$, which supplies nonlinguistic information $I$ relevant to the reciprocal's interpretation, just in case the set $\mathcal{S}_{c}$ has a member that entails every other one, where $\mathcal{S}_{c}=\{p \mid p$ is an interpretation of $\mathrm{S}$ obtained by interpreting the reciprocal as one of the nine quantifiers in (31), and $p$ is consistent with $I\}$. In that case, the use of $S$ in $c$ expresses the logically strongest proposition in $\mathcal{S}_{c}$.

It is stated that the SMH exploits contextually given information to give the literal meaning of a sentence. Then, depending on a sentence or a context, a different information can be input for the SMH. In what follows, the possible information input is listed, especially those used for the cases we discussed in this paper.

- Logical property of the relation, i.e. the scope of RECIP: symmetricity, transitivity etc.

- The group antecedent/the denotation of N-bar of a quantified antecedents:

(43) a. The highway exits are spaced five miles from each other.

\footnotetext{
${ }^{10}$ Note that our account use only one mode (BC), and an apparently different mode involving summing of small sets like (i) or (ii) is a by-product of particular RECIPS which are closed under union.

(i) $Q(A, \bigcup\{X \subseteq A \mid \operatorname{RECIP}(X, R)\})$

or equivalently

$$
\text { (ii) } Q(A,\{x \mid \exists Y \subseteq A(x \in Y \wedge \operatorname{RECIP}(Y, R))\})
$$

In van der Does (1993), this mode is proposed as one way to combine collectives with quantified subjects. Considering that reciprocals hold of a group, it could be natural to use the same mode for both cases. And Kamp and Reyle (1992) implicitly argued for this mode for reciprocals with quantified antecedents. As a matter of fact, this mode is equivalent to (BC) if RECIP is definable using TOT $\backslash I$. However, it cannot cover the whole range of the data; therefore, we contend that nothing is achieved with this additional mode.
} 
b. John's favorite bars are within a mile of each other.

- The determiner of a quantified antecedent

- Adjuncts:

(44) He and scores of other inmates slept on foot-wide wooden planks stacked atop each other - like sardines in a can - in garage-sized holes in the ground.

- Tense and aspect:

(45) The people in this room have been married to each other.

As Ray Jackendoff pointed out to us, statement (45) has stronger truth conditions than (18).

- Physical constraints: People (magazines) have only two sides. Vertebrae array in one line. One stack of bunk beds cannot fill a garage-size holes. Things cannot grind without touching.

- Encyclopedic knowledge: People get measles only once. People can waltz with only one person at a time. Countless stones.

- Social restrictions: Monogamy

- Typical (human) behavior in a particular situation: People (pirates) do not move at surprise, so stare a person in front of them. One stack of tables is enough to escape. People do/can not judge those who they don't know.)

- The assumptions in context of utterance:

(46) The inspector found peach fruit flies at four different locations within a mile of each other.

This statement would mean that each of the locations is within a mile of every other location, in a context where it was clear that the inspector was systematically criss-crossing the area he was searching. But in a context where it was clear that the inspector was making a linear sweep through a large area to collect a sample of fruit fly traps, the statement would mean that no two consecutive finds of fruit flies were more than a mile apart.

The SMH applies more generally than just to reciprocals, though it is formulated narrowly here. SMH is a semantic principle determining the literal meaning of utterances of certain expressions in any context appropriate for the expression. As employed here, the SMH is not a pragmatic principle - for example, for listeners to use in divining which reading of an ambiguous expression a speaker might intend on a given occasion. It does not concern how the speaker's meaning can diverge from literal meaning of an utterance. Thus its generality should not be expected to be the kind associated with pragmatic principles such as Grice's. 
If, for instance, donkey pronouns are ambiguous between a 'strong' and a 'weak' reading, and listeners use pragmatic principles to work out which meaning the speaker has in mind, the SMH cannot be one of the principles used to do this. When a donkey pronoun has as antecedent a $\uparrow$ MON $\uparrow$ quantifier, the 'strong' reading ("beat every donkey they own") is logically stronger than the 'weak' reading; but the weak reading is the one people actually get (Kanazawa (1994)).

(47) Some farmers who own a donkey beat it.

And with $\uparrow$ MON $\downarrow$ quantifiers as antecedent, the 'weak' reading ("beat some donkey he owns") is logically stronger, but the 'strong' reading is the one people actually get.

(48) Not every farmer who owns a donkey beats it.

Another case besides reciprocals, however, where the SMH does operate semantically to explain variation in literal meaning is presuppositions of implicative verbs. Coleman (1971) observes that the presupposition of, e.g., manage varies with context, as (49), (50) and (51) show.

(49) a. John managed to cash the check.

b. John tried to cash the check.

(50) a. He managed to run up a room service bill for $\$ 80,000$.

b. It is difficult to run up a room service bill for $\$ 80,000$.

(51) a. Our neighbors managed to schedule their wild party of the year the night before my German exam.

b. It is unlikely that our neighbors would schedule their wild party of the year the night before my German exam.

These cases have progressively weaker presuppositions, and each has has as its presupposition the strongest candidate consistent with information supplied in the context of utterance. A single sentence like (52) can have any of the three presuppositions, depending on context.

(52) Harry's dog manages to wake him up whenever he dozes off on the couch.

\section{New Predictions}

The SMH predicts that sentence

(53) Their bunk beds are on top of one another.

discussed by Kański (1987), means IAO rather than SR even though the antecedent group consists of just two members - which is correct. ${ }^{11}$

The SMH makes an interesting prediction regarding statements with nonincreasing quantifiers like (55).

\footnotetext{
${ }^{11}$ This successful prediction of the SMH is unfortunately accompanied by an incorrect one, however, namely that any reciprocal sentence will mean IAO if all stronger candidates are unsatisfiable. For instance, the SMH predicts that
} 
(55) Its members are so class conscious that few have spoken to each other, lest they accidentally commit a social faux pas.

The reciprocal in (55) means IAO. It can be explained by the following fact.

Fact 3. For monotone decreasing $Q$, if $\operatorname{RECIP}_{1} \Rightarrow \mathrm{RECIP}_{2}$, then $(\mathrm{BC})\left[Q, \operatorname{RECIP}_{2}\right] \Rightarrow(\mathrm{BC})\left[Q, \operatorname{RECIP}_{1}\right]$, provided $\mathrm{Q}$ is logical or $\mathrm{RECIP}_{2}$ is closed under union (e.g., is definable using TOT $\backslash I$ ).

The strength of various RECIPS is reversed with monotone decreasing quantifiers, and thus IAO is the strongest definition. As this meaning is not contradicted by any assumptions in given context, the reciprocal in (55) is correctly predicted to mean IAO.

The same accounts apply for the following two examples.

(56) She said she found other competitors on the women's circuit shallow, with closed minds, elaborating: No one even chats to each other.

(57) You know one or two Georges. You've met the occasional Cynthia. But none of them have ever lived together, or even know each other. You think it's unlikely they could have met up and got married behind your back.

For the non-monotone quantified antecedent of (58), none of the propositions that $\mathrm{BC}$ produces by combining the nine different interpretations of RECIP with the antecedent quantifier is stronger than any other - without additional information supplied by the context.

(58) The shop lined up four or five copies of a magazine against each other along a shelf, so the title appears to be a fast seller - a common US practice.

Utterances of (58) are natural only in special sorts of context. For instance, (58) would be felicitous in a context where it is understood that only a single connected group of copies of the magazine are next to each other. In this context, the strength ordering of RECIP is preserved under (BC) even for non-monotone quantifiers. Thus the SMH predicts that in such a context, (58) will mean that a set of 4 or 5 copies of a magazine satisfies IR for the relation of being lined up against, because the stronger definition $\mathrm{SR}$ is inconsistent. These observations support the conclusion that the SMH should assert that a reciprocal sentence can be uttered felicitously only in a context where there is a unique strongest interpretation consistent with the contextually given assumptions.

The following minimal pair shows how different contextual assumptions accompanied with relations can affect the truth conditions of reciprocal statements, and that the SMH correctly predicts the difference.

(59) Exactly 30 people know each other.

(60) Exactly 30 people are waltzing with each other.

(54) \# Those two people are each other's mother.

is true just in case one of the people is the mother of the other. In fact, contrary to the SMH, this sentence means Strong Reciprocity and is necessarily false. These examples suggest the need to revise the SMH in a way that preserves the prediction that the reciprocal means the strongest possible candidate but drops the insistence that only satisfiable candidates are possible. 
Consider these statements in the same situation: Several clubs are jointly holding a party, and there are 30 people altogether in the room. People of the same club know each other but they do not know other club members. Everyone is waltzing with his/her own partner. In this situation, we judge (59) false, but (60) true. Nothing contradicts with SR for the case of the relation of knowing. Then, the largest closed group (BC) seeks is the set of people in which everyone knows everyone else. It would be the largest club, which cannot have exactly 30 members since several clubs altogether have 30 members. Hence the statement is false in the given situation. On the other hand, with the assumptions in context of waltz, the strongest meaning is $\operatorname{SmR}(=W R, I A O)$. It is weaker than SR (=[2]) and $\mathbb{R}(=[1], \mathrm{LAR})$. As people cannot waltz with more than one person at the same time, SR and $\mathbb{R}$ cannot hold, hence $\mathrm{SmR}$ is the strongest meaning not contradicted in the given situation. Since $\mathrm{SmR}$ is closed under union (or definable with TOT $\backslash I$ ), the largest set (BC) seeks is the the collection of pairs who are waltzing with each other, which consists of exactly 30 people. Hence the statement is true in the situation.

\section{Further Context-Dependency of the Reciprocal}

(61) Parents evaluate their children - and students judge themselves and each other - on the basis of their ability to score well.

(62) The charm bracelet, with scores of tiny trinkets clanging against each other, also matches the noisy exuberance of the postwar era that created them.

(63) Two major theories about the Venusian surface are providing the geologists and geophysicists with intriguing puzzles: One holds that the surface of Venus consists of vast plates whose movements may resemble the tectonic plates of Earth's crust, which grind against each other or dive beneath one another to produce earthquakes, ignite volcanoes and shove masses of crustal rock into high mountains such as the Andes and the Himalayas.
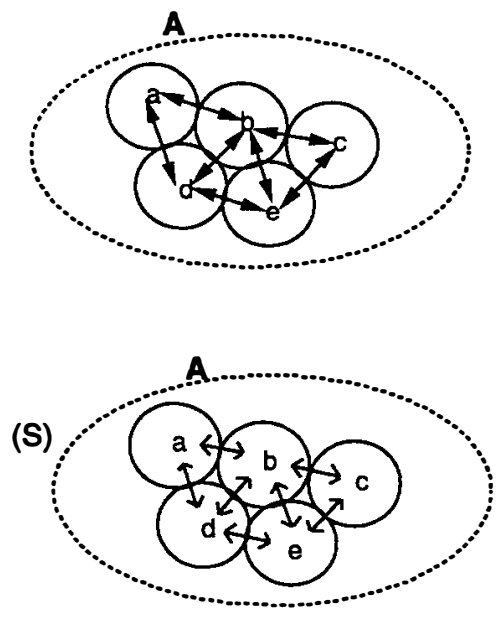


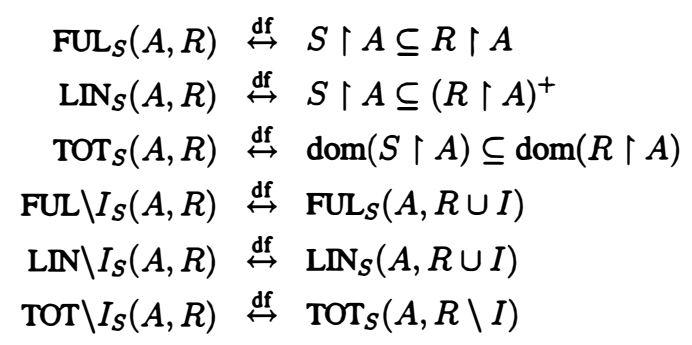

Fact 4. $Q_{S}^{\sharp}$ is monotone decreasing in $S$ for $Q^{\sharp}=$ FUL $\backslash I, \operatorname{LIN} \backslash I$, and TOT $\backslash I$. That is, if $S_{1} \subseteq S_{2}$ and $Q_{S_{2}}^{\sharp}(A, R)$, then $Q_{S_{1}}^{\sharp}(A, R)$.

Fact 5. The first two 'dimensions' of contextual variation in RECIP cannot be eliminated in favor of the new third 'dimension' of variation because, for instance, LIN $\backslash I$ cannot be defined in terms of FUL $\backslash I_{S}$, and so IR cannot be either. (cf. Sauerland (1994)

\section{Acknowledgments}

This work was supported in part by National Science Foundation grant number SBR93-19371, and also supported by the Information Technology Promotion Agency, Japan, as part of the R\&D of Basic Technology for Future Industries "New Models for Software Architecture" sponsored by NEDO (New Energy and Industrial Technology Development Organization). We are grateful to participants in the project on Cognitive Study of Situatedness in English and Japanese, sponsored by MONBUSHO.

\section{References}

Coleman, Linda. 1971. The case of vanishing presuppositions. In Proceedings of $B L S$ 1, pages 78-89.

Dalrymple, Mary, Makoto Kanazawa, Sam Mchombo, and Stanley Peters. 1994. What do reciprocals mean. In Mandy Harvey and Lynn Santelmann, editors, Proceedings of SALT IV, pages 61-78, Ithaca, NY. Cornell University.

Higginbotham, James. 1980. Reciprocal interpretation. Journal of Linguistic Research, 1(3):97-117.

Kamp, Hans and Uwe Reyle. 1992. From discourse to logic: An introduction to model theoretic semantics of natural language, formal logic and Discourse Representation Theory. MS, University of Stuttgart.

Kanazawa, Makoto. 1994. Weak vs. strong readings of donkey sentences and monotonicity inference in a dynamic setting. Linguistics and Philosophy, 17(2):109-158.

Kański, Zbigniew. 1987. Logical symmetry and natural language reciprocals. In Proceedings of the 1987 Debrecen Symposium on Language and Logic, pages 49-68, Budapest. Akadémiai Kiadó. 
Langendoen, D. Terence. 1978. The logic of reciprocity. Linguistic Inquiry, 9(2):177-197.

Moltmann, Friederike. 1993. Exception sentences and polyadic quantification. MS UCLA.

Roberts, Craige. 1987. Modal Subordination, Anaphora, and Distributivity. Ph.D. thesis, University of Massachusetts at Amherst.

Sauerland, Uli. 1994. Codistributivity and reciprocals. In MIT Working Papers 25. MIT.

Schwarzschild, Roger. 1992. Types of plural individuals. Linguistics and Philosophy, 15:641-675.

van Benthem, Johan. 1989. Polyadic quantifiers. Linguistics and Philosophy, 12(4):437-464.

van der Does, Jaap. 1993. Sums and quantifiers. Linguistics and Philosophy, pages 509-550. 